\title{
Probing extrasolar planet atmospheres through transits
}

\author{
Ignas Snellen \\ Institute for Astronomy, University of Edinburgh, Blackford Hill, Edinburgh EH9 3HJ, UK \\ and
}

Sterrewacht Leiden, Postbus 9513, 2300 RA, Leiden, The Netherlands

email: snellen@strw.leidenuniv.nl

\begin{abstract}
A revolution is taking place in the research of extra-solar planets with the discovery of the first exoplanets only a decade ago to the more than 100 systems known to date. Almost all of these extrasolar planets have been discovered using the radial velocity technique. Unfortunately, this limits the amount of information which can be obtain from these systems, with a $\sin i$ ambiguity in the planet's mass, and no further measurements of fundamental parameters as long as these planets can not be detected directly. This situation is very different in the rare case that the orbit of a planet has an inclination such that it occults its host star, as in the case of HD 209458b. Not only can the mass and radius of the planet be accurately determined, but it makes the system also suitable for many detailed follow-up studies, in particular atmospheric transmission spectroscopy. This has resulted in the detection of the atmosphere of HD 209458b in Sodium, and the discovery of an evaporating exosphere in Hydrogen, Carbon and Oxygen using the Hubble Space Telescope. In this paper I briefly review transiting exoplanets and methods to probe their atmospheres, with the emphasis on a new method of transmission spectroscopy making use of the Rossiter effect, which may be more suitable for the large ground-based telescopes.
\end{abstract}

\section{The extra-solar planet revolution}

The many talks during this conference about the $18^{\text {th }}$ and $19^{\text {th }}$ century voyages to observe the transits of Venus, show that it must have been a very exciting time to be an astronomer. In a few hundred years from now people may think in the same way about the early $21^{\text {st }}$ century, at least from a scientific perspective, with a genuine revolution taking place in extra-solar planet research.

We still virtually know nothing about other solar systems: what are the characteristics of extra-solar planets? How many planets are typical? What are their masses, compositions, orbits, and does their occurence depend on properties of the host star or certain environmental effects? However, it is expected that we will be able to answer many of these questions within the next few decades. Much of the driving force behind the boom in this area of research is the prospect of finding earth-like planets within our lifetimes, including possible evidence for carbon-based life.

Formation processes of stars and planets are so complex that they are almost impossible to derive from first principles. The developments of more and more powerful telescopes, instruments, and observation techniques are therefore crucial. For obvious reasons, direct detection of extra-solar planets is highly challenging and, so far, beyond reach. However, indirect methods have already been very successful, in particular the radial velocity technique which measures the reflex motion of a star about the star-planet barycentre (for extensive reviews on detection techniques see Perryman 2000; Sackett 1999). The first exoplanets around solar-type stars were discovered using this method almost a decade ago (Mayor \& Queloz 1995; Marcy \& Butler 1996), and more than 100 systems are known to 


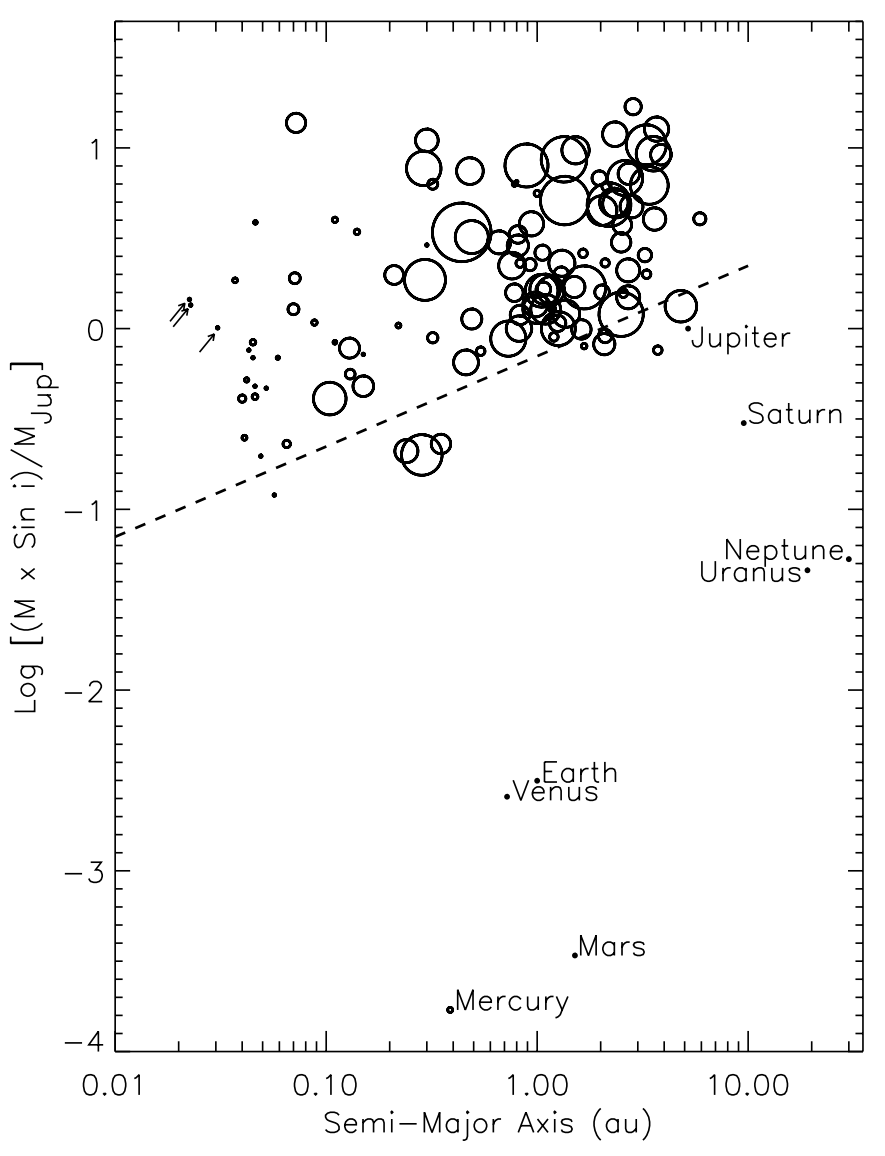

Figure 1. Current status of the extra-solar planet population discovered around main sequence stars, with orbital semi-major axis on the $\mathrm{x}$-axis and mass on the $\mathrm{y}$-axis (data from http://www.obspm.fr/encycl/encycl.html). The symbol sizes reflect the eccentricity of the orbits. Note that a large fraction of the exoplanet population discovered so far have highly eccentric orbits. The arrows indicate those planets discovered by their transits.

date (http://www.obspm.fr/encycl/encycl.html). An overview of the masses and orbits of the planets currently known is shown in Fig. 1. The limited data available to date have already resulted in several surprises. Firstly, the existence of the so-called Hot Jupiters, planets with orbital periods of only a few days, was not anticipated. Secondly, the fact that a high fraction of the orbits are strongly eccentric was also not expected. In addition it has been found that on average the stars hosting planets have a significantly higher metal content than the average solar-type star in the solar neighbourhood (Gonzales \& Laws 2000). These early unexpected results show that the predictive power of physical theory in this area of research has indeed so far been rather limited.

Although the Doppler wobble technique has been a great success, it also has its limitations. Since the inclinations of the planetary orbits are unknown, the amplitude of a radial velocity wobble strictly only results in a lower limit to the planetary mass, and not much else can be learned about the basic parameters of the exoplanet. A great advance could be made if starlight reflected from the planets were to be detected. The phasefunction of this signal depends on the orientation of the orbit and could therefore solve 
the $\sin i$ ambiguity. The detection of reflected light, even from Hot Jupiters, is, however, highly challenging, with expected contrasts of $10^{-4}$ to $10^{-5}$, or less. Deep spectroscopic observations have resulted in typical upper limits in geometric albedo of $<20 \%$, implying that these Hot Jupiters only possess a fraction of the reflectivity of Jupiter (e.g. Leigh et al. 2003). There is good hope that observations with the MOST satellite will yield the first positive results (see Matthews et al., these proceedings).

\section{Transiting exoplanets}

The Doppler wobble technique has been very successful in providing a large database of extra-solar planets. Unfortunately, detailed investigations of these systems individually can not yet be undertaken. This situation is very different in the rare case that the orientation of the orbit is such that the planet transits its host star. Not only can the mass and radius of the exoplanet be accurately determined, the transit itself is also suitable for many detailed follow-up studies. During transit, a Jupiter-size planet would block off in the order of $\sim 1 \%$ of the starlight, a factor of $\sim 100$ more than Venus. Unfortunately, the chances for this to happen are slim. From a random position in space, the chance that a planet in our solar system would transit the Sun ranges from about $1 \%$ for Mercury, to $0.1 \%$ for Jupiter. Fortunately, for Hot Jupiters this chance is more like $10 \%$.

To date, 14 Hot Jupiters with an orbital radius of $a<0.05$ have been discovered using the Doppler technique, and indeed one, HD 209458b, has been found to transit its host star (Charbonneau et al. 2000; Henry et al. 2000; Mazeh et al. 2000). It blocks off about $1.6 \%$ of the star-light for a period of $\sim 3 \mathrm{hr}$, every $3.52 \mathrm{~d}$. The combination of radial velocity data and accurate photometric observations with the HST have resulted in a precise determination of the fundamental parameters of HD 209458b, showing it has a mass of $0.69 \mathrm{M}_{\mathrm{Jup}}$ and radius of $1.35 \mathrm{R}_{\mathrm{Jup}}$ (Brown et al. 2001), and proving beyond any doubt that this is indeed a planet.

\subsection{Extra-solar planet transmission spectroscopy}

One of the great successes in recent astronomy has been the detection of an atmosphere around HD 209458b. This has been achieved using transmission spectroscopy. At particular wavelengths, corresponding to transitions of common atoms or molecules in the planetary atmosphere, the photometric transit is stronger due to extra absorption of star-light in the outer layers of the planetary atmosphere. Therefore, by measuring the transit signal as function of wavelength the atmospheric constituents can be determined. Atmospheric transmission spectroscopy was first used for a particular type of binary stars, $\zeta$ Aurigae, where the chromospheric structure of a large, cool star is studied when it eclipses its small companion (e.g. Wilson \& Abt 1954). This technique has also been used extensively for the planets in our own solar system, in particular using spacecraft, such as the Voyager I \& II, that observe the setting or rising of the sun or a star behind the limb of a planet (Smith \& Hunten 1990).

Detailed models indicated that the dominant features in the transmission spectra of Hot Jupiters are the Sodium and Potassium lines in the optical, and Helium and the $\mathrm{CO}, \mathrm{H}_{2} \mathrm{O}$ and $\mathrm{CH}_{4}$ molecules in the near infrared (e.g. Seager \& Sasselov 2000; Brown 2001; Hubbard et al. 2001). In addition, it was predicted that the close-in Hot Jupiters would have an extended exosphere in the form of a cometary tail, caused by the strong irradiation of the host star (Schneider et al. 1998).

It was Charbonneau et al. (2002) who first detected the atmosphere of HD 209458b in Sodium, by monitoring 6 transits with the Hubble Space Telescope. They found that in a region of $12 \AA$ around the two Sodium D lines the transit depth was $0.023 \pm 0.0057 \%$ deeper 

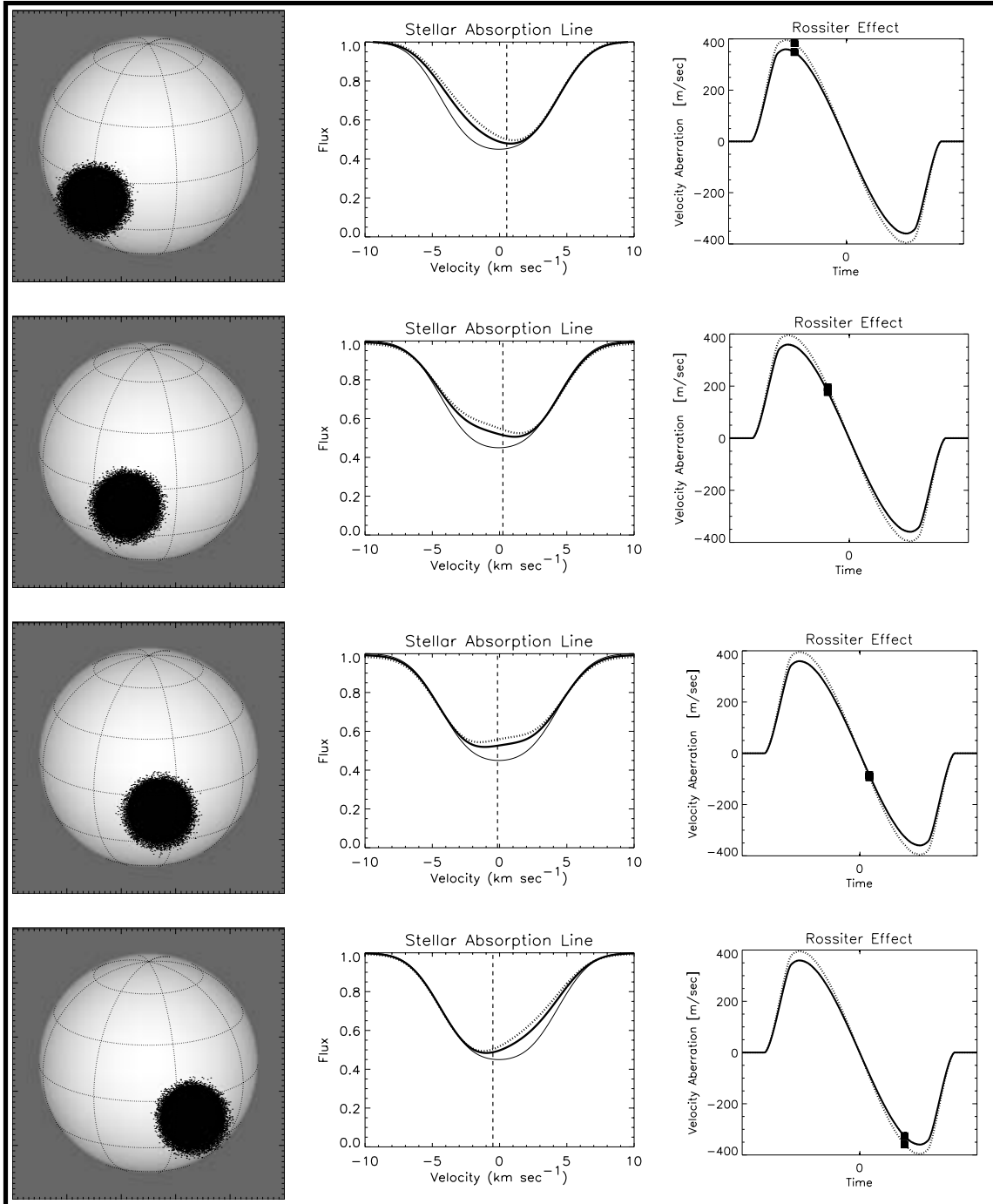

Figure 2. Illustration of the Rossiter effect, caused by a dark object transiting a rotating star. Since the object subsequently blocks off the approaching and receding parts of the stellar surface, a change in the profile of the rotationally broadened stellar absorption line occurs, which results in an effective red and blue shifting of the line. The perturbed and unperturbed absorption line profiles are shown in the middle panels by the thick and thin solid lines respectively. The resulting Rossiter effect is shown in the panels to the right. The effects of extra absorption caused by a possible atmosphere is indicated by the dotted lines. Note that for illustrative purposes the transiting object is significantly larger than HD 209458b.

than in the surrounding continuum. HST observations in the ultraviolet subsequently measured very strong absorption features, at a level of $5-10 \%$, from Hydrogen, Oxygen, and Carbon (Vidal-Madjar et al. 2003, 2004). The depth and width of these features suggest that this absorption comes from an evaporating exosphere of HD 209458b.

Attempts to detected the atmosphere or exosphere using ground-based telescopes have so far been unsuccessful. Observations targeting the Sodium D feature in the optical have 
resulted in upper limits of typically $1-2 \%$ (Brown et al. 2000; Bundy \& Marcy 2000; Moutou et al. 2001). Similar results have been obtained for the main absorption features in the near-infrared (Brown et al. 2002; Moutou et al. 2003). More stringent upper limits have been obtained in the infrared by Harrington (2002) using the secondary eclipse, by comparing the light from the star plus planet with that of the star only when the planet is occulted.

The main reason why ground-based observations do not yet reach the required precision seems to be the fact that atmospheric transmission spectroscopy relies on the comparison of spectra on and off transit, with the weather and instruments being insufficiently stable over timescales much greater than an hour.

\section{A new method of transmission spectroscopy}

We have developed a new method for probing the atmospheres of transiting exoplanets, making use of the Rossiter effect (Snellen 2004), which may be more suitable for groundbased telescopes.

\section{The Rositter effect}

The Rossiter effect was first observed for the binary star $\beta$ Lyrae, thereby showing that stars rotate (Rossiter 1924). If an object transits a rotating star, it will subsequently block off light from the approaching and receding parts of the stellar surface (Fig. 2). The resulting effect is an apparent red and blueshift of the rotationally broadened stellar absorption lines during the transit. Rossiter's observations showed this effect in $\beta$ Lyrae to have an amplitude of $10-15 \mathrm{~km} \mathrm{~s}^{-1}$. Schneider (1999) suggested that the Rossiter effect could also be observable for transiting exoplanets, and this was observed during a transit of HD 209458b by Queloz et al. (2000). The exact radial velocity pattern can constrain the relative orientation of the orbit. As expected, in the HD 209458 system the the planetary orbit is in the same direction as the stellar rotation, with a measured upper limit to the angle between the orbital plane and equatorial plane of the star of $30^{\circ}$.

The amplitude of the Rossiter effect, determined by Queloz to be $\sim 35-40 \mathrm{~m} \mathrm{~s}^{-1}$, is mainly dependent on the rotation velocity of the host star and the relative size of the planetary disk. Due to this latter dependence, this effect can be used to probe the atmosphere of HD 209458b in the same way as with conventional transmission spectroscopy. At particular wavelengths the amplitude of the Rossiter effect will be higher due to the extra absorption in the planetary atmosphere, causing an increase in the effective size of the planetary disk. The main advantage of using the Rossiter effect over a straightforward photometric signal is that it only relies on the relative positions of the stellar lines in the same spectra, and is therefore much less hampered by calibration issues. Particularly from the ground this means that potentially a much higher accuracy could be achieved.

The resulting excess in the Rossiter effect depends on the width and depth of the atmospheric absorption feature. If the absorption in Sodium were to be homogeneous over the $12 \AA$ wavelength range used by Charbonneau et al. (2002), it would result in an excess in the Rossiter effect in the Sodium D lines of $\sim 0.5 \mathrm{~m} \mathrm{~s}^{-1}$ compared to other stellar lines. If the absorption is peaked at smaller scales it could lead to an excess of up to $\sim 2 \mathrm{~m} \mathrm{~s}^{-1}$ (Snellen 2004).

\section{Results from UVES archival data}

The new method was tested using an archival dataset from the UV-Visual Echelle Spectrograph (UVES) on the Very Large Telescope (VLT). The data comprised 4 on-transit datasets, centered on the Sodium D absorption lines at 5990 and $5996 \AA$, with $15 \times 400$-s 

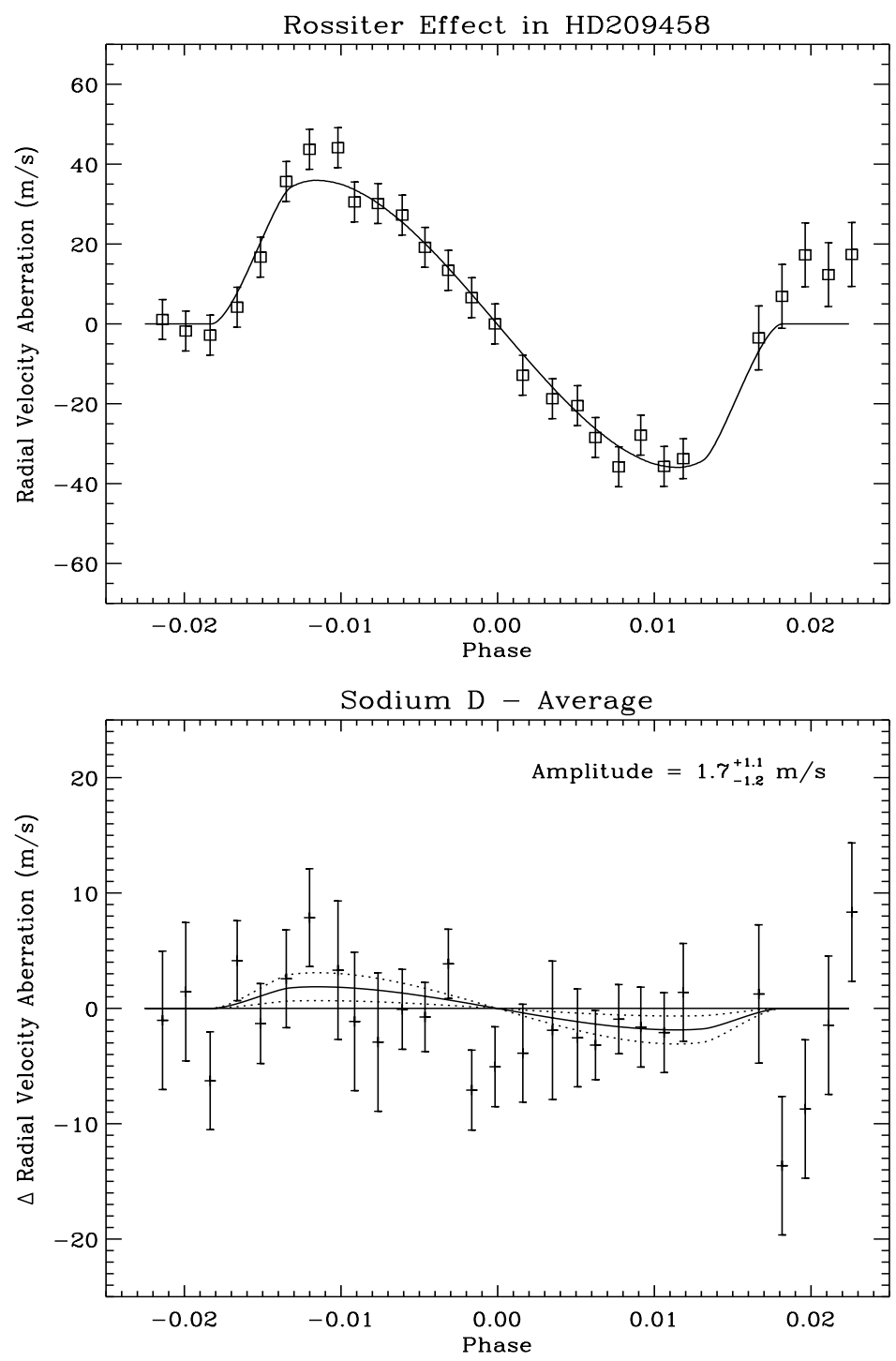

Figure 3. Upper panel: The Rossiter effect as observed for HD 209458 using an archival UVES/VLT dataset covering 4 transits. Lower panel: The relative excess in the Rossiter effect for the two Sodium D lines. The $1 \sigma$ confidence limits are indicated by the dotted lines. The best-fit model shows an excess of $1.7_{-1.2}^{+1.1} \mathrm{~m} \mathrm{~s}^{-1}(1.4 \sigma)$.

exposures covering each transit. Unfortunately, a significant fraction of the transit time was spent for this method on unnecessary calibration stars, and in most cases only a part of the transit was observed. This has significantly reduced the accuracy.

The reduction and analysis of the data are discussed in detail in Snellen (2004). A typical positional accuracy of $\sim 1 \times 10^{-4} \AA$ (0.006 pixel) was obtained for the strongest 
lines. This corresponds to a precision in relative radial velocity of $\sim 5 \mathrm{~ms}^{-1}$ for each exposure. The combined dataset of $4 \times 15$ exposures was binned and fitted with a model for the Rossiter effect, which was constructed by numerically simulating the transit of a dark object over a limb-darkened star. The best fitting model shows an excess in the Rossiter effect of $1.7_{-1.2}^{+1.1} \mathrm{~m} \mathrm{~s}^{-1}(1.4 \sigma-$ Fig. 3$)$. Assuming a homogeneous absorption over the width of the stellar line, this corresponds to a photometric signal using conventional atmospheric transmission spectroscopy of $7 \pm 5 \times 10^{-4}$. Although this archival dataset is far from ideal, it shows that for ground-based observations the use of the Rossiter effect instead of a photometric signal results in an order of magnitude improvement in precision. This, while a further factor $2-3$ in accuracy can be gained for observations specifically designed for this method, covering complete transits without unnecessary gaps for calibration purposes.

\section{Future prospects}

Plans for further observations of HD 209458 are in place to explore the full potential of the Rossiter effect. Since observations of complete transits are necessary to reach the highest possible precision, only $6-7$ transits per year are optimally timed for observatories in the Northern hemisphere, and only half of that for those in the south. Obvious absorption features to target in the optical are the Sodium and Potassium lines, for which with the improved accuracy we would expect a firm detection. Comparison of a detection using the Rossiter method, which is most sensitive to atmospheric absorption on a sub-Ångstrom scale, with the conventional photometric method as achieved with the HST, could for the first time constrain the profile of the Sodium absorption feature. Also, the so-far illusive Helium feature at $10830 \AA$ (e.g. Moutou et al. 2003) will be targeted. Not only has it been predicted to be one of the planet's strongest atmospheric features at a level of $\sim 0.25 \%$ (Seager \& Sasselov 2000), Helium is also expected to be a major component in the planet's extended exosphere, with the potential to significantly boost the absorption signal to an excess in the Rossiter effect of $5-15 \mathrm{~m} \mathrm{~s}^{-1}$. The He I feature itself is relatively weak in solar type stars, and also a tracer of coronal activity with a patchy surface distribution, which may result in some rotational velocity jitter. However, fortunately just $2.5 \AA$ blueward of it is the deep stellar absorption line of Si I, within the width of the expected atmospheric absorption, and centered on the expected evaporating exosphere of HD 209458b.

\section{Finding new transiting exoplanets}

So far HD 209458b is the only realistic target for atmospheric transmission spectroscopy. The three exoplanets discovered recently in the OGLE-III survey (Udalski 2002a,b) are all transiting much fainter stars (OGLE-TR-56b, OGLE-TR-113b and OGLE-TR-132b; Konacki et al. 2003, 2004; Bouchy et al. 2004). However, in the light of probing exoplanet atmospheres using the Rossiter effect, finding new planets transiting bright stars would be very exciting. Transiting planets around rapidly rotating stars and/or late M stars would be of particular interest. Firstly, the faster a star rotates, the larger the Rossiter effect, and therefore the larger the possible effect of an atmosphere. Secondly, for late-type $M$ stars with a low enough surface temperature the near-infrared molecular absorption features in the planet's atmosphere, such as $\mathrm{H}_{2} \mathrm{O}, \mathrm{CO}$ and $\mathrm{CH}_{4}$, will start to cover many stellar absorption lines. Since the measurement of the Rossiter effect could then be combined over many stellar lines, again a significant increase in precision could be achieved. Note that a Jupiter-sized planet transiting a rapidly rotating M dwarf (say with $v=10-20 \mathrm{kms}^{-1}$ ), would produce a Rossiter effect with an amplitude of 
$1-2 \mathrm{~km} \mathrm{~s}^{-1}$ ! Potentially, with the strength and profiles of the main atmospheric absorption lines can not only the chemical composition be determined, but also constraints be put on planetary rotation and temperature structure, cloud properties and winds in the upper atmosphere.

A very promising survey to find such planets is SuperWASP. Conducted by a consortium of UK institutions it aims at the robotic operation of up to 8 ultra-wide-field cameras, each with a field of view of $7.8 \times 7.8$, to obtain well sampled light curves of a large sample of stars in the magnitude range $\sim 7$ to 13 at better than $1 \%$ precision (Street et al. 2003).

\section{References}

Brown T.M., Butler R.P., Charbonneau D., Noyes R.W., Sasselov D., Libbrecht K.G., Marcy G.W., Seager S., Vogt S.S., 2000, 197th AAS Meeting, 11.05; BAAS, Vol. 32, p.1417

Brown T.M., Charbonneau D., Gilliland R.L., Noyes R.W., Burrows A., 2001, ApJ 552, 699

Brown T.M., 2001, ApJ 553, 1006

Brown T.M., Libbrecht K.G., Charbonneau D., 2002, PASP 114, 826

Bouchy F., Pont F., Santos N., Melo C., Mayor M., Queloz D., Udry S., 2004, A\&A, 421, L13

Bundy K.A., Marcy G.W., 2000, PASP 112, 1421

Charbonneau D., Brown T.M., Latham D.W., Mayor M., 2000, ApJ 529, 45

Charbonneau D., Brown T.M., Noyes R.W., Gilliland R.L., 2002, ApJ 568, 377

Gonzales G. Laws C, 2000, AJ 119, 390

Harrington J., Deming D., Matthews K., Richardson L.J., Rojo P., Steyert D., Wiedemann G., Zeehandelaar D., 2002, 201st AAS Meeting, 46.04; BAAS, Vol. 34

Henry G.W., Marcy G.W., Butler R.P., Vogt S.S., 2000, ApJ 529, L41

Hubbard W.B., Fortney J.J., Lunine J.I., Burrows A., Sudarsky D., Pinto P., 2001, ApJ 560, 413

Leigh C., Collier Cameron, A., Udry, S., Donati, J., Horne, K., James, D., Penny, A., 2003, MNRAS, 346, L16

Konacki M., Torres G., Sasselov D., Pietrzynski G., Udalski A., Jha S., Ruiz M.-T., Gieren W., Minniti D., 2004, ApjL 609, L37

Konacki M., Torres G., Jha S., Sasselov D., 2003, Nature, 421, 507

Marcy G.W., Butler R.P., 1996, ApJ 464, L147

Mayor M., Queloz D., 1995, Nature, 378, 355

Mazeh T., Naef D., Torres G., Latham D.W., Mayor M., Beuzit J.-L., Brown T., Buchlave L., Burnet M., Carney B.W. et al. 2000, ApJ 532, L55

Moutou C., Coustenis A., Schneider J., St Gilles R., Mayor M., Queloz D., Kaufer A., 2001, A\&A 371, 260

Moutou C., Coustenis A., Schneider J., Queloz D., Mayor M. 2003, A\&A 405, 341

Sackett, P. 1999, in "Planets Outside the Solar System: Theory and Observations." Eds J.-M. Mariotti and D. Alloin. Dordrecht ; Boston : Kluwer Academic Publishers, p.189

Perryman, M. 2000, Rep. Prog. Phys., 63, 1209

Queloz, D., Eggenberger, A., Mayhor, M., Perrier, C., Beuzit, J.L., Naef, D., Sivan, J.P., Udry, S., 2000, A\&A, 359, L17

Rossiter, R.A., 1924, AJ, 60, 15

Seager S., Sasselov D.D., 2000, ApJ 537, 916

Schneider J., Rauer H., Lasota J.P., Bonazzola S., Chassefiere E., 1998, in "Brown dwarfs and extrasolar planets", eds R. Rebolo, E.L. Martin; M.R. Zapatero Osorio, ASP Conference Series 134, 241

Schneider J., 1999, in VLT opening symposium, Antofagasta, ed. Paresce F., Springer, p. 499

Smith G., Hunten D., 1990, Reviews of Geophysics, vol. 28, p. 117

Snellen I.A.G. 2004, MNRAS, MNRAS, 353, L1

Street R., D.L. Pollacco, A. Fitzsimmons, F.P. Keenan, Keith Horne, S. Kane, A. Collier Cameron et al., 2003, in "Scientific Frontiers in Research on Extrasolar Planets", ASP 
Conference Series, Vol 294, Eds. Drake Deming and Sara Seager. (San Francisco: ASP) p. 405-408

Udalski A, Zebrun K., Szymanski M., Kubiak M., Soszynski I., Szewczyk O., Wyrzykowski L., Pietrzynski G., 2002a, Acta Astronomica, 52, 115

Udalski A, Szewczyk O., Zebrun K., Pietrzynski G., Szymanski M., Kubiak M., Soszynski I., Wyrzykowski L., 2002b, Acta Astronomica, 52, 317

Vidal-Madjar A, Lecavelier des Etangs A., Désert J.-M., Ballester G.E., Ferlet R., Hébrard G., Mayor M., 2003, Nature 422, 143

Vidal-Madjar A, Désert J.-M., Lecavelier des Etangs A., Hébrard G., Ballester G.E., Ehrenreich D., Ferlet R., McConnell J.C., Mayor M., Parkinson C.D., 2004, ApJ, 604, L69

Wilson O., Abt H., 1954, ApJS 1, 1

\section{Discussion}

Mikhail MARov: In searching for Earth-like like planets, do you think - just based on our current knowledge - that in a configuration completely different from what we have for the solar system the closely-located planets to the star would allow the preservation of Earth-like planets? Would they disturb the whole situation near the mother star so tremendously that there would be no chance at all for survival of an Earth-like planet?

IGNAS SNellen: No, I really don't think that. Yet, if you have a hot Jupiter, then it would be very unlikely that you will find an Earth-like planet. But that is only a reasonably small fraction of the total extra-solar planet population.

JAYMie MatThews: Ignas, does your model include the tidally-locked rotation of the planet's atmosphere, as well, to order of magnitude. I'm assuming there's something like a $\mathrm{km} / \mathrm{s}$, or so, in the atmosphere in both directions, and the diameter of the planet relative to the star is large enough that there's actually a gradient in the projected radial velocity of the star. Will that blur out the signal, and is that part of your calculation?

IGNAS Snellen: Do you mean: would the planetary absorption be too narrow in wavelength?

JAYMie MatTheWs: No. What I'm saying is that the planet is rotating, so the absorbing - let's say for the sake of argument the sodium - atmosphere has the trailing edge moving towards you and the leading edge moving away with about a $\mathrm{km} / \mathrm{s}$, and the equatorial velocity of the star is maybe 10 , or so, $\mathrm{km} / \mathrm{s}$, so it's not insignificant in terms of the effects and the absorption in the radial velocity parameter space. I am just curious if that's part of the calculation.

Ignas Snellen: No. At this moment I haven't concentrated on these kinds of models, and how broad you would expect the actual absorption to be. The only thing I can say is that if you just take the face value of HST observations, and just would expect exactly the same absorption for this object, then you would expect an amplitude of about 30 $\mathrm{cm} / \mathrm{s}$, or so. What I measure here is $1.7 \mathrm{~m} / \mathrm{s}$, so it's much higher. However, this is what I would expect because the HST signal is averaged over $11 \AA$, while this Rossiter effect is basically within the absorption of the sodium line which is smaller than $1 \AA$, so I would really expect that secondary Rossiter effect would be a factor of 5 or so larger - say on the $\AA$ scale - than it is at the actual HST scale, but the exact details are not really clear.

Fritz Benedict: A comment: While you rightly point out that all of these planets have been discovered by radial velocity techniques, there's at least been one measured confirmed to be a planetary mass - and this was through astrometry with the Hubble 
Space telescope (Gliese 876). The connection here is that we had to remove the parallax with exquisite precision in order to see the wobble due to the planet.

MARY BRÜCK: Did you observe yesterday's Transit of Venus? Is somebody doing it with this technique that you are using?

Ignas Snellen: No, I don't think so, but the actual Rossiter effect is very well known. But to actually do this for Venus would be much more difficult because you have to measure the total spectrum of the sun and to do this ...

MARY BRÜCK: Well, I would have thought this would have been a jolly good test, wouldn't it after all?

IGNAS SNELLEN: No, the actual amplitude you expect for Venus - what is it? a 1000 times smaller? a mm/s? So I don't think it's going to be observed, but it may be interesting; maybe in eight years I will be doing that.

MARY BRய̈CK: Yes, good.

FRITZ BenEDICT: There's actually a group in Hawaii who were looking at the moon yesterday to see the effect of the Transit of Venus on the integrated light of the sun from the moon, so it's not the Rossiter effect, but at least they were looking for a transit!

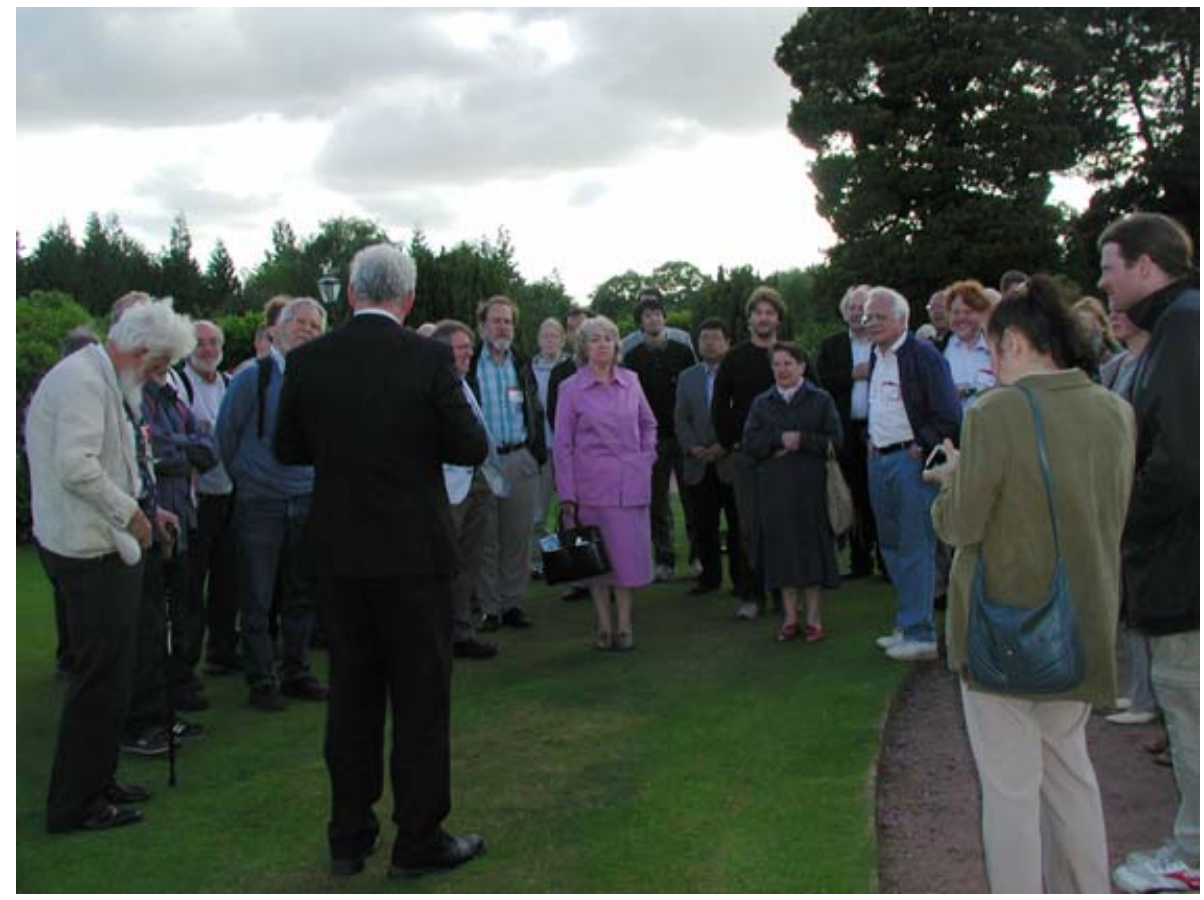

Stonyhurst College Observatory tour 